\title{
Long-range dynamical magnetic order and spin tunneling in the cooperative paramagnetic states of the pyrochlore analogous spinel antiferromagnets $\mathrm{CdYb}_{2} \mathrm{X}_{4}(X=\mathrm{S}$ or Se)
}

\author{
P. Dalmas de Réotier, ${ }^{1}$ C. Marin, ${ }^{1}$ A. Yaouanc, ${ }^{1}$ C. Ritter, ${ }^{2}$ A. Maisuradze, ${ }^{3}$ B. Roessli, ${ }^{4}$ A. Bertin, ${ }^{1}$ P. J. Baker,,${ }^{5}$ and A. Amato ${ }^{6}$ \\ ${ }^{1}$ Univ. Grenoble Alpes, CEA, INAC/PHELIQS, F-38000 Grenoble, France \\ ${ }^{2}$ Institut Laue-Langevin, Boite Postale 156X, F-38042 Grenoble Cedex 9, France \\ ${ }^{3}$ Department of Physics, Tbilisi State University, Chavchavadze 3, GE-0128 Tbilisi, Georgia \\ ${ }^{4}$ Laboratory for Neutron Scattering and Imaging, Paul Scherrer Institute, CH-5232 Villigen-PSI, Switzerland \\ ${ }^{5}$ ISIS Facility, STFC Rutherford Appleton Laboratory, Chilton, Didcot, OX11 0QX, United Kingdom \\ ${ }^{6}$ Laboratory for Muon-Spin Spectroscopy, Paul Scherrer Institute, CH-5232 Villigen-PSI, Switzerland
}

(Received 16 May 2017; revised manuscript received 10 August 2017; published 2 October 2017)

\begin{abstract}
Magnetic systems with spins sitting on a lattice of corner sharing regular tetrahedra have been particularly prolific for the discovery of new magnetic states for the last two decades. The pyrochlore compounds have offered the playground for these studies, while little attention has been comparatively devoted to other compounds where the rare earth $R$ occupies the same sublattice, e.g., the spinel chalcogenides $\operatorname{Cd} R_{2} X_{4}(X=\mathrm{S}$ or Se). Here, we report measurements performed on powder samples of this series with $R=\mathrm{Yb}$ using specific heat, magnetic susceptibility, neutron diffraction, and muon-spin-relaxation measurements. The two compounds are found to be magnetically similar. They long-range order into structures described by the $\Gamma_{5}$ irreducible representation. The magnitude of the magnetic moment at low temperature is 0.77 (1) and 0.62 (1) $\mu_{\mathrm{B}}$ for $X=\mathrm{S}$ and Se, respectively. Persistent spin dynamics is present in the ordered states. The spontaneous field at the muon site is anomalously small, suggesting magnetic moment fragmentation. A double spin-flip tunneling relaxation mechanism is suggested in the cooperative paramagnetic state up to $10 \mathrm{~K}$. The magnetic space groups into which magnetic moments of systems of corner-sharing regular tetrahedra order are provided for a number of insulating compounds characterized by null propagation wave vectors.
\end{abstract}

DOI: 10.1103/PhysRevB.96.134403

\section{INTRODUCTION}

The study of geometrical frustration, where the crystal geometry prevents individual interactions from being satisfied, is one of the central themes in condensed matter research. Following the rich phase diagrams discovered for the insulating pyrochlore compounds $R_{2} M_{2} \mathrm{O}_{7}$, where $R$ is a trivalent rare-earth ion and $M=\mathrm{Ti}$ and $\mathrm{Sn}$ [1,2], there is a strong momentum to look at other compounds crystallizing within the same cubic crystal structure, but with different $M$ tetravalent elements. For example, reports have been presented for $M=\operatorname{Zr}$ [3-8], Hf [9-11], Pb [12,13], Pt [14-16], and Ge [12,16-20].

The crystal electric fields acting on the $R$ ions for these compounds [21] are expected to be rather similar. This is understandable since the ion geometry arrangement around them is the same. Interestingly, there is another family of compounds for which the $R$ ions also sit on a lattice of corner-sharing regular tetrahedra: the cadmium chalcogenide spinels [22]. They are of particular interest because the point symmetry $(\overline{3} m)$ at the $R$ site is the same as in the pyrochlores but the coordination and bonding around $R$ with the $\mathrm{O}^{2-}$ neighboring ions forming a nearly regular octahedron is very different (see Fig. 1). Therefore their crystal-electric fields (CEFs) should be substantially different. This is confirmed by the appearance of a spin-ice state in $\mathrm{CdEr}_{2} \mathrm{Se}_{4}$ [24] and possibly in $\mathrm{CdEr}_{2} \mathrm{~S}_{4}$ [25], and its absence in $\mathrm{CdHo}_{2} \mathrm{~S}_{4}$ [26]. This is in contrast to the pyrochlore titanates or stannates for which the spin ice state is observed when $R=$ Ho, whereas the $R=$ Er systems have planar local anisotropy. Therefore the spinels offer the possibility to extend the study of the lattice of corner sharing $R$ tetrahedra to unknown territory thanks to different CEFs.
Here, we report an investigation of the insulating spinel chalcogenides $\mathrm{CdYb}_{2} X_{4}$ with $X=\mathrm{S}$ or Se performed on powder samples using specific heat, magnetic susceptibility, neutron diffraction, and muon-spin-relaxation ( $\mu \mathrm{SR})$ measurements. Compared to the study performed by Higo and collaborators for the same compounds [27], we determine their magnetic structure and present a characterization of their spin dynamics using $\mu \mathrm{SR}$. In contrast to the pyrocholore ytterbium stannate and titanate, which are splayed ferromagnets [28,29], the two ytterbium spinels are antiferromagnets with magnetic moments perpendicular to their local threefold axis. However, the observed paramagnetic spin tunneling is rather similar to previous findings for $\mathrm{Yb}_{2} \mathrm{Ti}_{2} \mathrm{O}_{7}, \mathrm{Yb}_{2} \mathrm{Sn}_{2} \mathrm{O}_{7}$, and $\mathrm{Nd}_{2} \mathrm{Sn}_{2} \mathrm{O}_{7}$ [30,31], pointing to the topology of the corner-sharing tetrahedra lattice as its origin.

\section{EXPERIMENTAL}

The synthesis of $\mathrm{CdYb}_{2} \mathrm{~S}_{4}$ and $\mathrm{CdYb}_{2} \mathrm{Se}_{4}$ was achieved in a two-step approach using sealed quartz ampoules. High purity starting materials $(5 \mathrm{~N})$ were used: ytterbium metal, sulfur, and $\mathrm{CdS}$, or selenium, and CdSe. The first step was the preparation of $\mathrm{Yb}_{2} \mathrm{~S}_{3}$ and $\mathrm{Yb}_{2} \mathrm{Se}_{3}$ by heat treatment up to $650{ }^{\circ} \mathrm{C}$. The second step consisted of grinding the mixture $\mathrm{CdS}$ and $\mathrm{Yb}_{2} \mathrm{~S}_{3}$ (or CdSe and $\mathrm{Yb}_{2} \mathrm{Se}_{3}$ ), then pressing it into pellets. The final solid state reaction leading to $\mathrm{CdYb}_{2} \mathrm{~S}_{4}$ and $\mathrm{CdYb}_{2} \mathrm{Se}_{4}$ was achieved by heating the pellets up to $900{ }^{\circ} \mathrm{C}$ for two weeks. The single phase nature of the obtained compounds was checked by x-ray powder diffraction. A more detailed discussion of the crystalline purity of the two samples will be presented in Sec. III B 1 . 

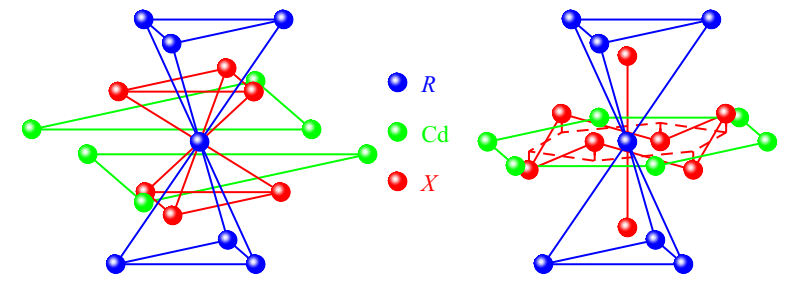

- $R$

- $M$

- 0

FIG. 1. Comparing the local environments at a rare-earth position for the spinel and pyrochlore compounds, on the left and right panels, respectively. Right-hand-side part of the figure reprinted with permission from Ref. [23]. Copyright 2011 by the American Physical Society.

The heat capacity and susceptibility measurements were performed with commercial instruments, namely the Physical Property Measurement System and the Magnetic Property Measurement System, both from Quantum Design, inc. The neutron diffraction measurements were conducted at the D20 high-intensity powder two-axis diffractometer of the Institut Laue Langevin, Grenoble, France. The muon spin rotation and relaxation measurements ( $\mu \mathrm{SR})$ were mostly performed at the MuSR spectrometer of the ISIS pulsed muon source, Rutherford Appleton Laboratory, Chilton, UK, and partly at the GPS spectrometer of the Swiss Muon Source, Paul Scherrer Institute, Villigen, Switzerland. Owing to the strong neutron absorption cross-section of $\mathrm{Cd}$, we used a hollow cylinder sample holder for the diffraction experiments: for each compound, $\approx 8 \mathrm{~g}$ of powder were inserted into the space available between the two coaxial cylinders of diameter 14 and $16 \mathrm{~mm}$.

\section{RESULTS}

\section{A. Macroscopic measurements}

In Fig. 2, we display the specific heat $C_{\mathrm{p}}$ in the whole temperature range. The results for the two compounds are rather similar, except above $\approx 7 \mathrm{~K}$ for which the thermal response is larger for $\mathrm{CdYb}_{2} \mathrm{Se}_{4}$. This is essentially explained by a sizably larger molar mass of this system, which shifts down its phonon spectrum relative to the other. This will

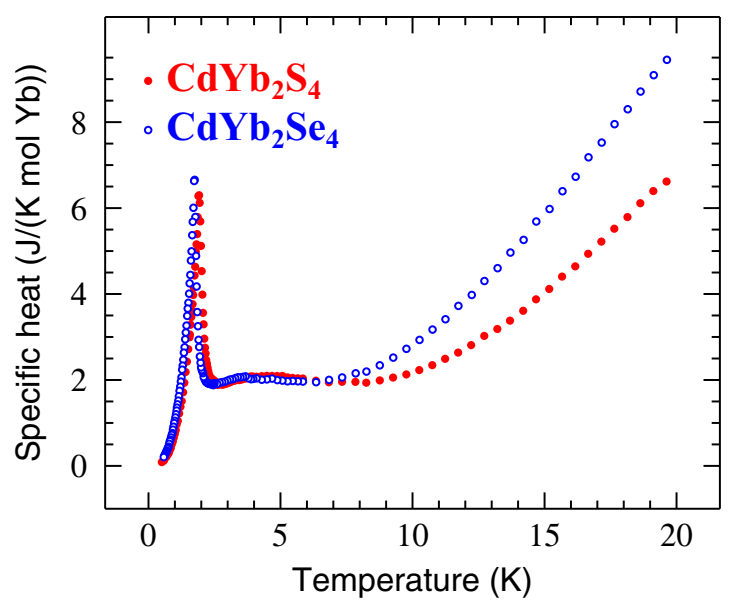

FIG. 2. Heat capacity of $\mathrm{CdYb}_{2} \mathrm{~S}_{4}$ and $\mathrm{CdYb}_{2} \mathrm{Se}_{4}$ vs temperature.

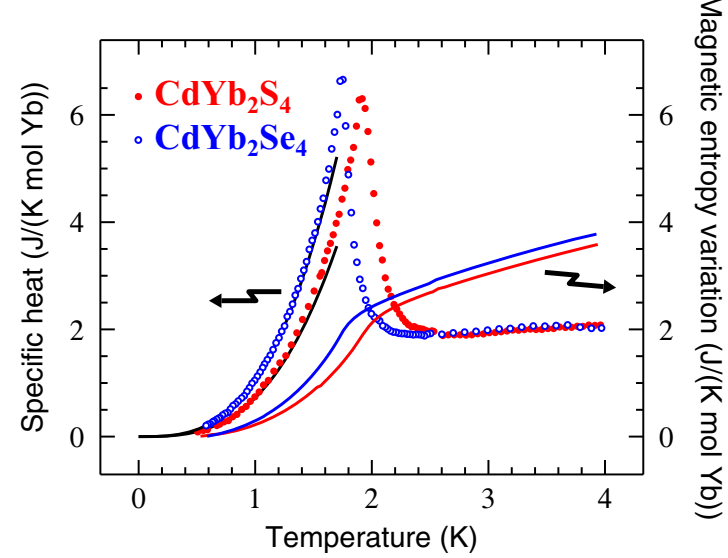

FIG. 3. Low-temperature part of Fig. 2. The black full lines result from a fit of the function $C_{\mathrm{p}}=\mathcal{B} T^{3}$ to the data below $1.2 \mathrm{~K}$, with $\mathcal{B}=0.73$ (3) and 1.06 (5) $\mathrm{J} \mathrm{K}^{-4} \mathrm{~mol}^{-1}$ for $\mathrm{CdYb}_{2} \mathrm{~S}_{4}$ and $\mathrm{CdYb}_{2} \mathrm{Se}_{4}$, respectively. The color full lines display the variation of magnetic entropy deduced from $C_{\mathrm{p}}(T)$.

not concern us here. Similar peaks are observed just below $2 \mathrm{~K}$ pointing out thermodynamic phase transitions. The peak shape suggests these transitions to be second order. We shall determine with neutron and $\mu \mathrm{SR}$ that they are of magnetic origin. Only mild bumps in $C_{\mathrm{p}}$ are observed between 2 and $6 \mathrm{~K}$. Hence short-range magnetic correlations in the correlated states are relatively weak in these compounds [32].

In Fig. 3, the low-temperature parts of $C_{\mathrm{p}}$ are shown. The critical temperatures $T_{\mathrm{c}}$ extracted from $C_{\mathrm{p}}$ are listed in Table I. Below $T_{\mathrm{c}}$ we find $C_{\mathrm{p}} \propto T^{3}$, a power law behavior expected for a conventional antiferromagnet. The spin wave velocities $v_{\mathrm{sw}}$, deduced from $C_{\mathrm{p}}$ following the method explained elsewhere [33], are given in Table I. Knowing $v_{\mathrm{sw}}$, the scale of the exchange integral $\mathcal{I}$ can be inferred [33]. We get $\mathcal{I} / k_{\mathrm{B}}=$ 0.40 (1) and 0.35 (1) $\mathrm{K}$ for the sulfide and the selenide, respectively. Here, $k_{\mathrm{B}}$ is Boltzmann's constant. In line with the similarity of $C_{\mathrm{p}}$, the variation of magnetic entropy (Fig. 3) follows the same trend for the two compounds, except for a temperature shift corresponding to the transition temperature difference. Between 0.5 and $4 \mathrm{~K}$, this variation approaches $4 \mathrm{~J} /(\mathrm{K}$ mol $\mathrm{Yb})$, i.e., $\approx 0.7 R \ln 2$ where $R$ is the ideal gas constant.

TABLE I. Different physical parameters resulting from this study. The quantities are the spin wave velocity $v_{\mathrm{sw}}$, the lattice parameter $a$, the position parameter $x$ for the chalcogen element (determined at 10 and $2.7 \mathrm{~K}$ for the sulfide and selenide, respectively), the critical temperature $T_{\mathrm{c}}$ of the magnetic transition derived from the heat capacity data, and the spontaneous moments at $100 \mathrm{mK}$. The units are given in the second row. The uncertainties are only of statistical origin.

\begin{tabular}{|c|c|c|c|c|c|}
\hline & $\begin{array}{c}v_{\mathrm{sw}} \\
\mathrm{m} \mathrm{s}^{-1}\end{array}$ & $\begin{array}{l}a \\
\AA\end{array}$ & $\begin{array}{l}x \\
-\end{array}$ & $\begin{array}{l}T_{\mathrm{c}} \\
\mathrm{K}\end{array}$ & $\begin{array}{c}m(T=100 \mathrm{mK}) \\
\mu_{\mathrm{B}}\end{array}$ \\
\hline $\mathrm{CdYb}_{2} \mathrm{~S}_{4}$ & $141(2)$ & $11.003(2)$ & $0.2594(5)$ & 1.92 & 0.77 (1) \\
\hline $\mathrm{CdYb}_{2} \mathrm{Se}_{4}$ & $130(2)$ & $11.455(2)$ & 0.2575 & 1.75 & $0.62(1)$ \\
\hline
\end{tabular}




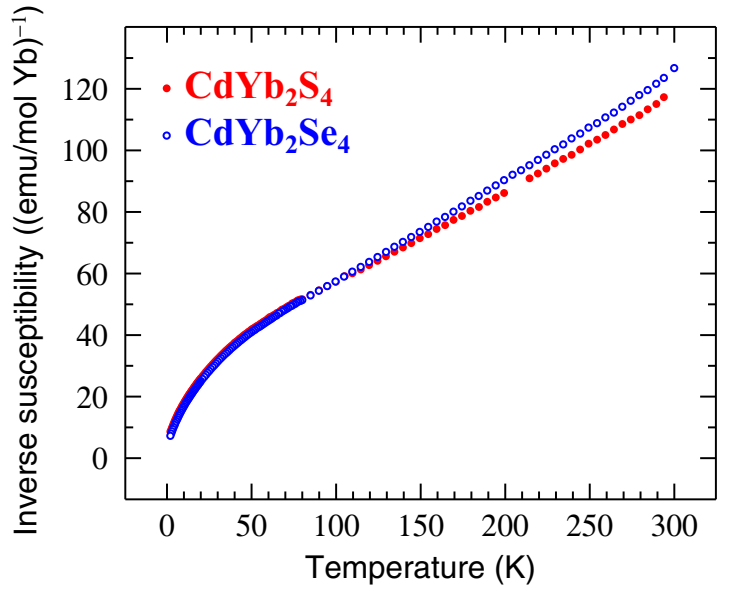

FIG. 4. Inverse susceptibility versus temperature for $\mathrm{CdYb}_{2} \mathrm{~S}_{4}$ and $\mathrm{CdYb}_{2} \mathrm{Se}_{4}$. The data have been recorded with an applied field of 0.9 and $1.5 \mathrm{mT}$, respectively. The fields are sufficiently low for the data to reflect the susceptibilities.

The inverse susceptibility data are collected in Fig. 4. The magnetic responses are rather similar, in particular at low temperatures. The expected straight line in a Curie-Weiss picture can be approximately found above $\approx 50 \mathrm{~K}$ and at low temperature. However, the Curie-Weiss temperature $\theta_{\mathrm{CW}}$ deduced from such analysis would depend on the temperature range at which the analysis is performed. In fact, the thermal behavior of the susceptibilities is strongly influenced by the crystal fields acting at the rare-earth ions [27]. We will therefore use the mean-field approximation formula $\mathcal{I}=3 k_{\mathrm{B}}\left|\theta_{\mathrm{CW}}\right| / z_{\mathrm{nn}} J(J+1)$ (see, e.g., Ref. [33]) to deduce $\left|\theta_{\mathrm{CW}}\right| \approx 13$ and $11 \mathrm{~K}$ for the sulfide and selenide, respectively. Here, $J=7 / 2$ is the spin of the $\mathrm{Yb}^{3+}$ manifold and $z_{\mathrm{nn}}=6$ is the number of nearest neighbors to a magnetic ion. The $\theta_{\mathrm{CW}}$ values are consistent with those found by Higo et al. With a ratio $f=\left|\theta_{\mathrm{CW}}\right| / T_{\mathrm{c}} \gtrsim 6$ for the frustration index [34], we conclude to the influence of frustration on the magnetic properties of these two spinels. Our bulk data clearly show that the two compounds have similar magnetic properties. Table I suggests the selenide to be slightly less magnetic than the sulfide. We now turn to the microscopic probe measurements, which reveal exotic magnetic properties.

\section{B. Microscopic probe measurements}

\section{Neutron diffraction results}

The crystallographic structure of the $\mathrm{CdYb}_{2} \mathrm{~S}_{4}$ and $\mathrm{CdYb}_{2} \mathrm{Se}_{4}$ spinels is described according to the $F d \overline{3} m$ cubic space group $[35,36]$. With the adopted description where the origin of the cubic unit cell is at a point of symmetry $\overline{3} \mathrm{~m}$, the $\mathrm{Cd}$ and $\mathrm{Yb}$ ions, respectively, occupy $8 \mathrm{a}$ and 16d Wyckoff positions of respective coordinates $\left(\frac{1}{8}, \frac{1}{8}, \frac{1}{8}\right)$ and $\left(\frac{1}{2}, \frac{1}{2}, \frac{1}{2}\right)$. The $\mathrm{S}$ or Se ions are located at a 32e position $(x, x, x)$. From our Rietveld neutron refinements using FULLPROF [37], we obtain the lattice parameters $a$ and the chalcogen position parameters $x$ as given in Table I.

Figures 5 and 6 display the diffraction diagrams recorded at $10 \mathrm{~K}$ for $\mathrm{CdYb}_{2} \mathrm{~S}_{4}$ and $2.7 \mathrm{~K}$ for $\mathrm{CdYb}_{2} \mathrm{Se}_{4}$, respectively. For both compounds the width of the Bragg peaks corresponds

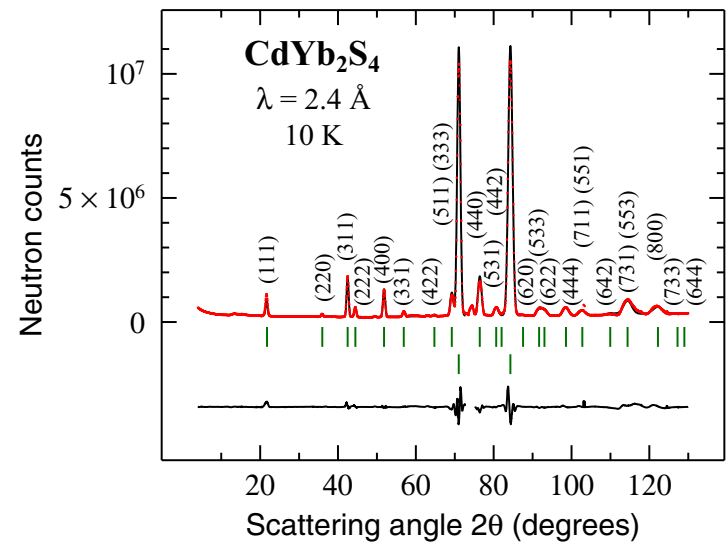

FIG. 5. Neutron diffraction pattern for a powder of $\mathrm{CdYb}_{2} \mathrm{~S}_{4}$ at $10 \mathrm{~K}$ vs the scattering angle $2 \theta$. Neutrons of wavelength $2.4 \AA$ were used. The experimental data are drawn as red dots. The solid line shows the result of a Rietveld refinement. The bottom line displays the difference between the experimental data and the refinement. The observed reflections are labeled with Miller indices. The vertical markers indicate the position of the expected Bragg peaks. The second row of markers corresponds to Bragg peaks from the $\mathrm{Cu}$ sample container.

to the instrument resolution. The structure refinements are very good. Only a peak at $\approx 74.5^{\circ}$ and perhaps two very tiny contributions at about $63^{\circ}$ and $97^{\circ}$ in the $\mathrm{CdYb}_{2} \mathrm{~S}_{4}$ data are not present in the model, suggesting the presence of a minority parasitic phase in this sample.

The magnetic scattering data deduced from the difference of the diffraction diagrams recorded at $100 \mathrm{mK}$ and $10 \mathrm{~K}$ for $\mathrm{CdYb}_{2} \mathrm{~S}_{4}$ and at $100 \mathrm{mK}$ and $2.7 \mathrm{~K}$ for $\mathrm{CdYb}_{2} \mathrm{Se}_{4}$ exhibit neutron intensity at the positions of the nuclear Bragg peaks. An additional narrow peak is observed at scattering angle

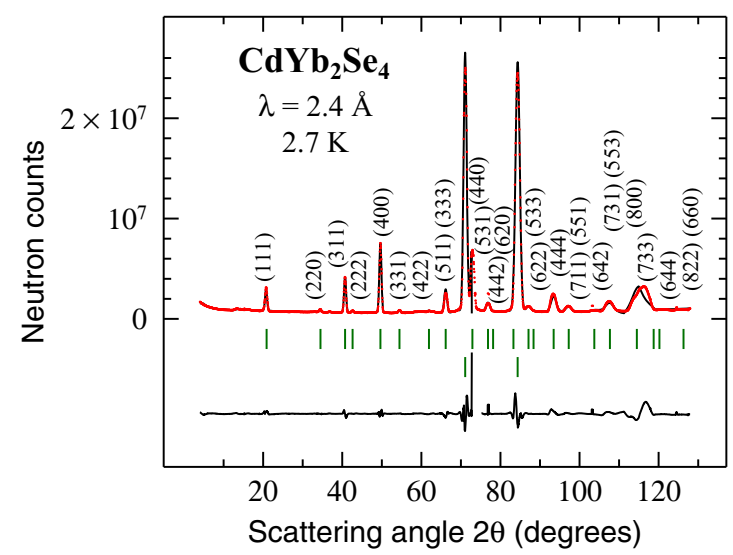

FIG. 6. Neutron diffraction pattern for a powder of $\mathrm{CdYb}_{2} \mathrm{Se}_{4}$ at $2.7 \mathrm{~K}$ vs the scattering angle $2 \theta$. Neutrons of wavelength $2.4 \AA$ were used. The experimental data are drawn as red dots. The solid line shows the result of a Rietveld refinement. The bottom line displays the difference between the experimental data and the refinement. The observed reflections are labeled with Miller indices. The vertical markers indicate the position of the expected Bragg peaks. The second row of markers corresponds to Bragg peaks from the $\mathrm{Cu}$ sample container. 


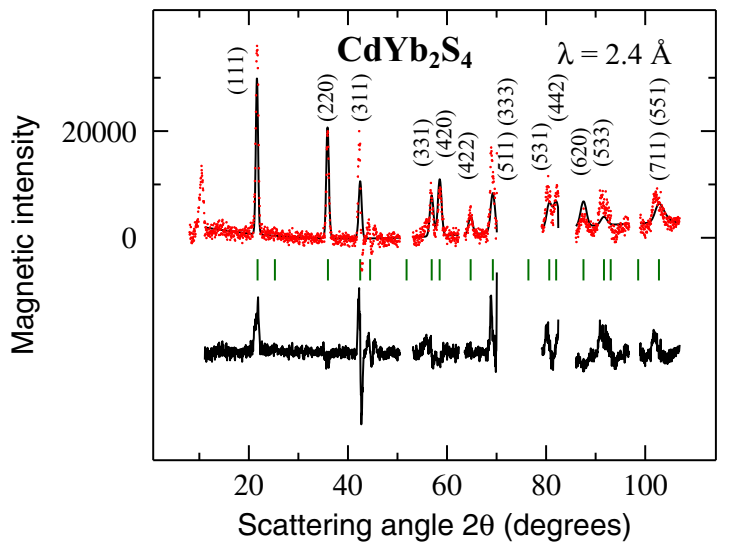

FIG. 7. Magnetic neutron scattering pattern of $\mathrm{CdYb}_{2} \mathrm{~S}_{4}$, i.e., difference between the diagrams recorded at $100 \mathrm{mK}$ and $10 \mathrm{~K}$. The fit according to the magnetic structure described in the main text is shown as a solid line. The bottom line displays the difference between the experimental data and the refinement. The vertical markers indicate the positions of the possible magnetic Bragg peaks while the observed reflections are labeled with Miller indices.

$2 \theta \approx 10.3^{\circ}$ and $10.1^{\circ}$ for the sulfide and selenide, respectively (Figs. 7 and 8). Relative to the other magnetic peaks, its intensity is somewhat stronger for the former compound. Remarkably, the peak observed in $\mathrm{CdYb}_{2} \mathrm{Se}_{4}$ could be indexed as $\left(\frac{1}{2}, \frac{1}{2}, \frac{1}{2}\right)$. This is not the case for the sulfide peak for which the $\left(\frac{1}{2}, \frac{1}{2}, \frac{1}{2}\right)$ peak would be shifted by $0.3-0.4^{\circ}$ compared to the observed peak. It would be tempting to assign these peaks at low angles to a structure with a magnetic propagation vector equal or close to $\mathbf{k}=\left(\frac{1}{2}, \frac{1}{2}, \frac{1}{2}\right)$. This idea suffers, however, from two strong reservations: (i) there are no further magnetic peaks which could be linked to this hypothetical propagation vector and (ii) some scattering intensity is still observed around $10^{\circ}$ in $\mathrm{CdYb}_{2} \mathrm{~S}_{4}$ at $2.5 \mathrm{~K}$, i.e., above the $T_{\mathrm{c}}$ value deduced from the heat capacity measurements and at

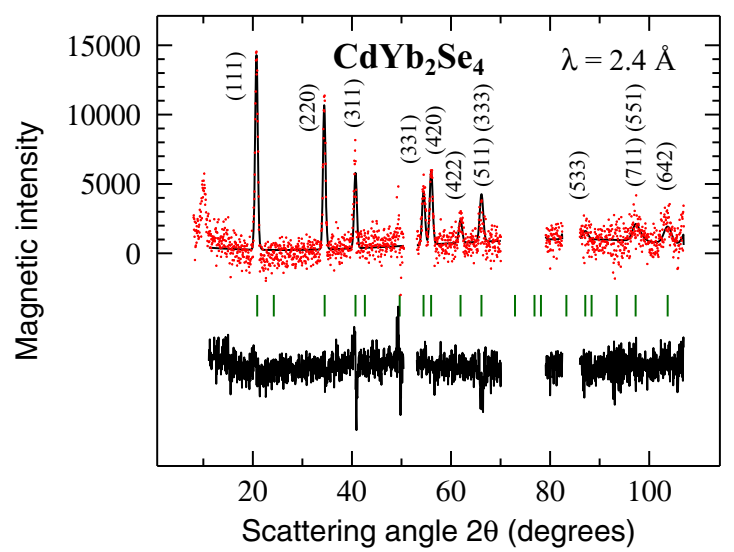

FIG. 8. Magnetic neutron scattering pattern of $\mathrm{CdYb}_{2} \mathrm{Se}_{4}$, i.e., difference between the diagrams recorded at $100 \mathrm{mK}$ and $2.7 \mathrm{~K}$. The fit according to the magnetic structure described in the main text is shown as a solid line. The bottom line displays the difference between the experimental data and the refinement. The vertical markers indicate the positions of the possible magnetic Bragg peaks while the observed reflections are labeled with Miller indices.

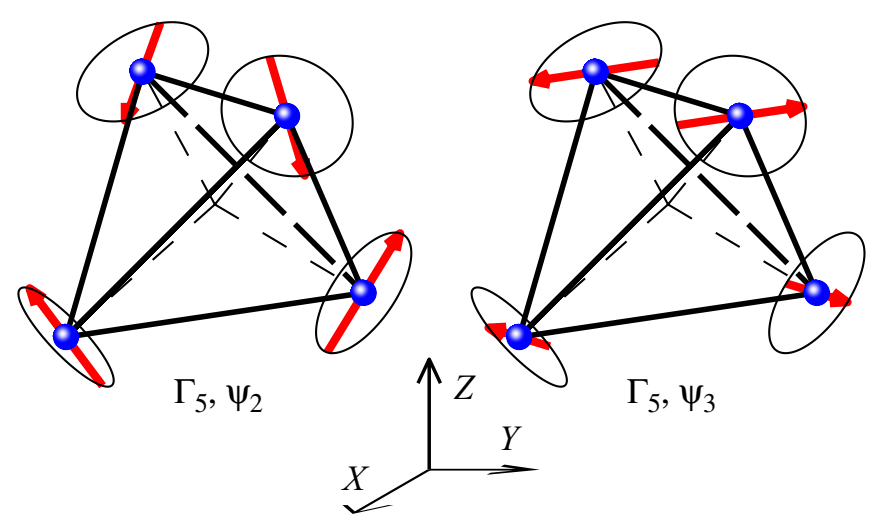

FIG. 9. Magnetic structures corresponding to the $\psi_{2}$ (left) and $\psi_{3}$ (right) modes of the $\Gamma_{5}$ irrep. The thin dashed lines represent the three-fold local symmetry axes. For both structures, the magnetic moments are confined to planes perpendicular to these axes. The cubic axes are denoted as $(X, Y, Z)$.

a temperature where all other magnetic peaks have vanished. These facts strongly suggest that the intensity observed around $10^{\circ}$ in both compounds arises from a long-range ordered magnetic parasitic phase present in the two compounds, a nuclear reflection of which is observed at $10 \mathrm{~K}$ and $74.5^{\circ}$ in the sulfide. For the analysis of the magnetic scattering data, only the intensity recorded above $11^{\circ}$ for the two systems will be considered.

The magnetic diffraction patterns are displayed in Figs. 7 and 8 for $\mathrm{CdYb}_{2} \mathrm{~S}_{4}$ and $\mathrm{CdYb}_{2} \mathrm{Se}_{4}$, respectively. Following the previous discussion, since the magnetic intensity of the $\mathrm{CdYb}_{2} \mathrm{~S}_{4}$ and $\mathrm{CdYb}_{2} \mathrm{Se}_{4}$ phases is only observed at the nuclear Bragg peak positions, we conclude that the magnetic structure propagation wave vector is $\mathbf{k}=(0,0,0)$. The magnetic order is long range since the width of the magnetic Bragg peaks corresponds to the diffractometer resolution. Following a representation analysis study for the symmetry at the rare-earth site, the possible magnetic structures are obtained from linear combinations of the basis vectors belonging to one of the four irreducible representations (irreps) labeled $\Gamma_{3,5,7,9}$ (see, e.g., Ref. [38]). From the presence of magnetic scattering for both systems at the (111) and (220) Bragg positions and its absence at (200), the $\Gamma_{3,7,9}$ irreps are excluded. Satisfactory Rietveld refinements of the experimental data (Figs. 7 and 8) are obtained with a magnetic structure belonging to the $\Gamma_{5}$ representation. This representation is two-dimensional with basis vectors denoted as $\psi_{2}$ and $\psi_{3}$. The magnetic structures arising from $\psi_{2}, \psi_{3}$, or any linear combination of them yield the same neutron diffraction pattern for a powder. For a reliable selection, measurements with polarized neutrons on crystals would be needed [39]. Powder measurements under an applied magnetic field are an alternative [40]. The common feature of $\psi_{2}$ and $\psi_{3}$ is that the magnetic moment at each rare earth is perpendicular to the local trigonal axis (Fig. 9).

Figures 10 and 11 display the temperature dependencies of the $\mathrm{Yb}^{3+}$ magnetic moment as determined by neutron diffraction. The magnetic moments at $100 \mathrm{mK}$ are listed in Table I. The magnetic moment moduli are somewhat reduced relative to $\approx 1 \mu_{\mathrm{B}}$ in the $\mathrm{Yb}_{2} \mathrm{Ti}_{2} \mathrm{O}_{7}$ and $\mathrm{Yb}_{2} \mathrm{Sn}_{2} \mathrm{O}_{7}$ pyrochlore compounds, illustrating the difference between 


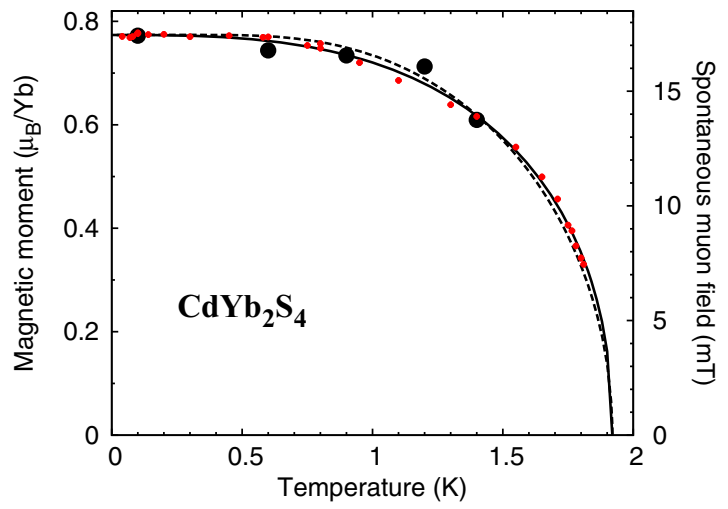

FIG. 10. Temperature dependence of the $\mathrm{Yb}^{3+}$ magnetic moment determined from neutron scattering (large black bullets) and the spontaneous field at the muon site (small red bullets) measured for $\mathrm{CdYb}_{2} \mathrm{~S}_{4}$. The error bars on the experimental data are smaller or equal to the symbol size. The full line is a fit of the phenomenological law $B_{0}\left[1-\left(T / T_{\mathrm{c}}\right)^{\alpha}\right]^{\beta}$ to the spontaneous field. The parameters are $B_{0}=17.46$ (4)mT, $\alpha=2.93$ (13) and $\beta=0.447$ (14). The dashed line shows the prediction of a mean-field model based on the $S=1 / 2$ Brillouin function.

the pyrochlores and the spinels. An understanding of this difference requires to determine the crystal-field ground state and the strength of the molecular field at the rare-earth site.

\section{2. $\mu \mathrm{SR}$ results}

In Fig. 12, we display asymmetry spectra recorded close to the magnetic transition for the two compounds. The detection of oscillations characteristic of spontaneous muon precession means that a compound is magnetically ordered at the temperature of observation [41]. It is clearly the case at 1.70 and $1.65 \mathrm{~K}$ for the selenide and $1.82 \mathrm{~K}$ for the sulfide. The $T_{\mathrm{c}}$ values from specific heat and the measured $\mu \mathrm{SR}$ spectra are

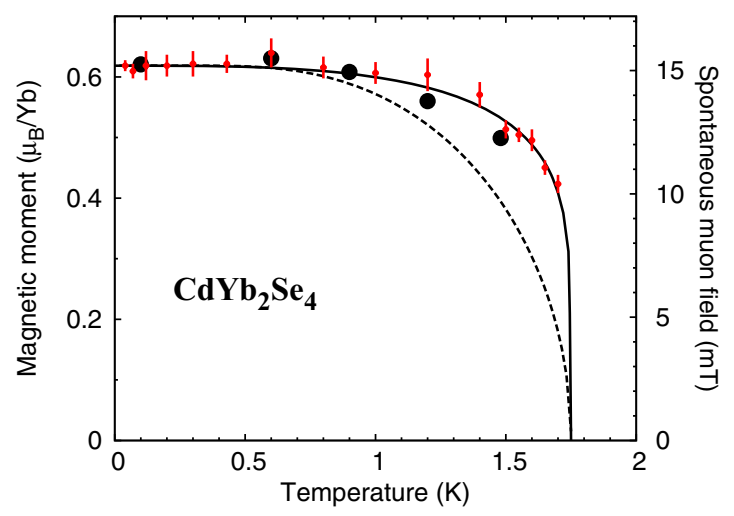

FIG. 11. Temperature dependence of the $\mathrm{Yb}^{3+}$ magnetic moment determined from neutron scattering (large black bullets) and the spontaneous field at the muon site (small red bullets) measured for $\mathrm{CdYb}_{2} \mathrm{Se}_{4}$. The error bars on the magnetic moment are smaller or equal to the symbol size. The full line is a fit of the same phenomenological law as in Fig. 10 to the spontaneous field with $B_{0}=15.23$ (9) $\mathrm{mT}, \alpha=3.2$ (6), and $\beta=0.17$ (2). The dashed line shows the prediction of a mean-field model based on the $S=1 / 2$ Brillouin function.

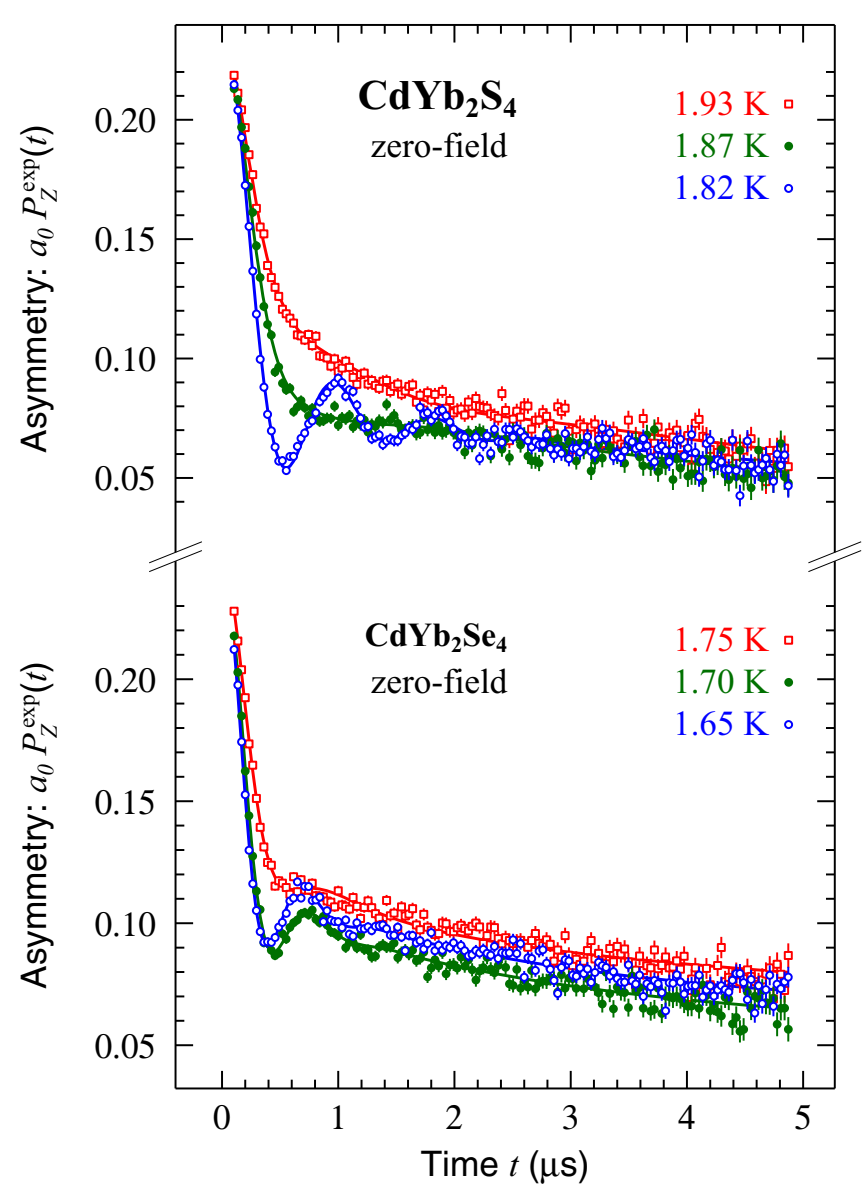

FIG. 12. Zero-field $\mu$ SR spectra recorded close to the magnetic transition in $\mathrm{CdYb}_{2} \mathrm{~S}_{4}$ (top) and $\mathrm{CdYb}_{2} \mathrm{Se}_{4}$ (bottom). The oscillations appear in a very narrow temperature range and are not overdamped. This is an indication for the good quality of the samples. It also suggests that critical dynamics does not play an important role.

consistent. The spontaneous field at the muon site is plotted versus temperature in Figs. 10 and 11, respectively, for the two compounds. The thermal variations deduced from neutron diffraction and $\mu \mathrm{SR}$ are consistent.

To conclude our discussion of the spectra below $T_{\mathrm{c}}$, we note the finite slopes at long time. They are in fact temperature independent down to far below $0.1 \mathrm{~K}$. The associated spinlattice relaxation rate is $\lambda_{Z} \approx 0.4 \mu \mathrm{s}^{-1}$ for the two compounds, a signature of the so-called persistent spin dynamics [30].

It has been recently shown that careful analyses of $\mu \mathrm{SR}$ spectra recorded in correlated paramagnetic regimes can yield useful information $[31,42]$. The spectra for the two compounds of interest here are best described as the weighted sum of two dynamical Kubo-Toyabe functions; see Fig. 13(a) for an illustration in the case of $\mathrm{CdYb}_{2} \mathrm{Se}_{4}$. Figure 13(b) displays the temperature dependence of the parameters extracted from a combined fit of the seven zero-field (ZF) spectra recorded for $\mathrm{CdYb}_{2} \mathrm{Se}_{4}$ : the relative population of one of the two muon sites $f_{1}$, the field widths $\Delta_{i}$ and the common inverse correlation time $v_{\mathrm{c}}$. Similar spectra (not shown) were obtained for $\mathrm{CdYb}_{2} \mathrm{~S}_{4}$. In fact, these results are reminiscent of those recently published for $\mathrm{Yb}_{2} \mathrm{Ti}_{2} \mathrm{O}_{7}, \mathrm{Yb}_{2} \mathrm{Sn}_{2} \mathrm{O}_{7}$, and $\mathrm{Nd}_{2} \mathrm{Sn}_{2} \mathrm{O}_{7}$ [31,42]: a temperature independent $v_{c}$ in the range of the 

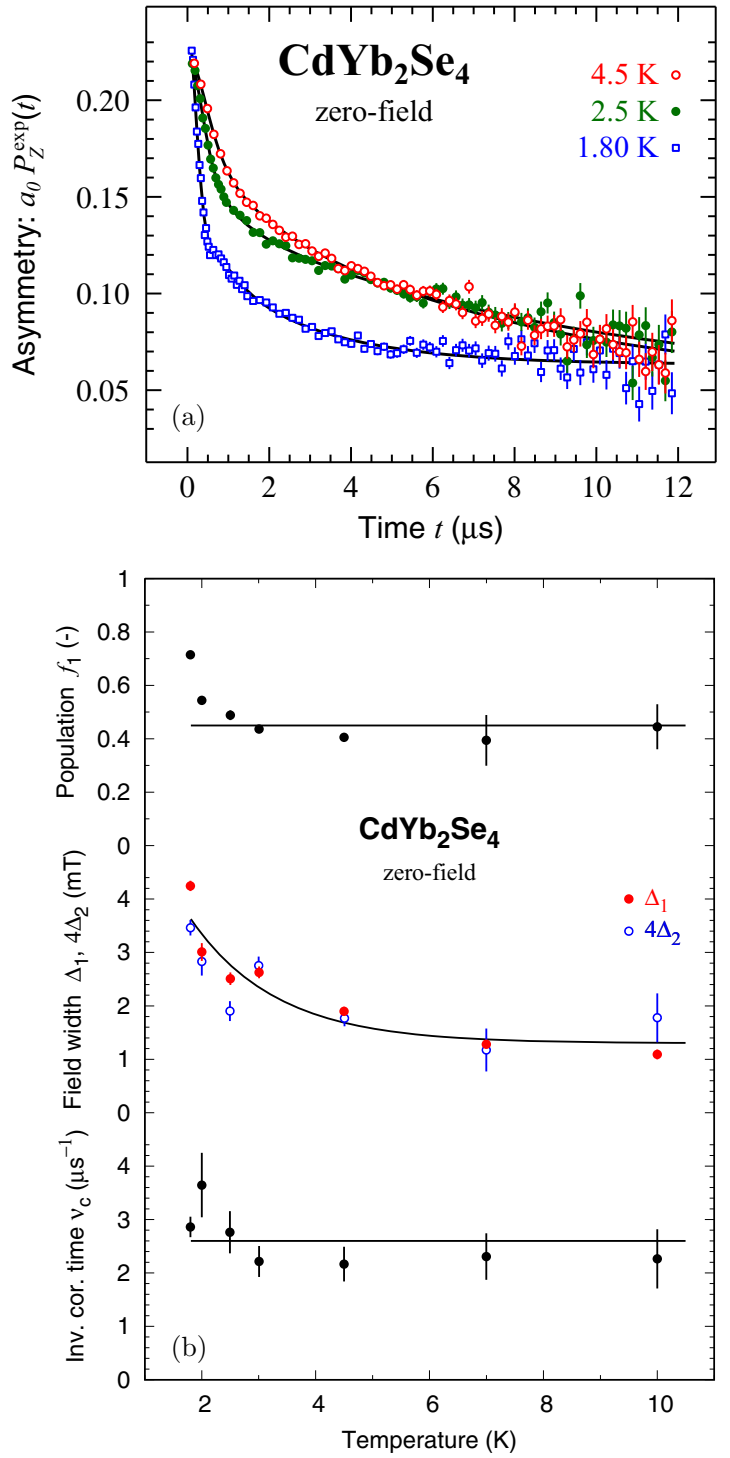

FIG. 13. (a) A selection of zero-field $\mu$ SR spectra recorded in the correlated paramagnetic phase of $\mathrm{CdYb}_{2} \mathrm{Se}_{4}$. Solid lines result from fits as described in the main text. (b) Thermal dependence of the fitting parameters. The full lines are guides to the eye.

inverse microsecond and an increase in field width as the compound is cooled to $T_{\mathrm{c}}$. At first sight, one could be surprised by the small $v_{\mathrm{c}}$ values. The strong effect of an external field as small as $10 \mathrm{mT}$ on the spectra is an additional proof of the quasi-static spin dynamics at play; see Fig. 14. As expected, the $f_{1}$ parameter is essentially found to be temperature independent, with the exception of the region close to $T_{\mathrm{c}}$, where features associated with the magnetic transition could not be grasped by the model.

\section{DISCUSSION}

We first stress that the results from Ref. [27] and ours compare favorably when comparison is possible, i.e., whenever data for a given technique are available from both studies. This means that samples of different origin have similar responses. The two compounds of the current study show a very similar

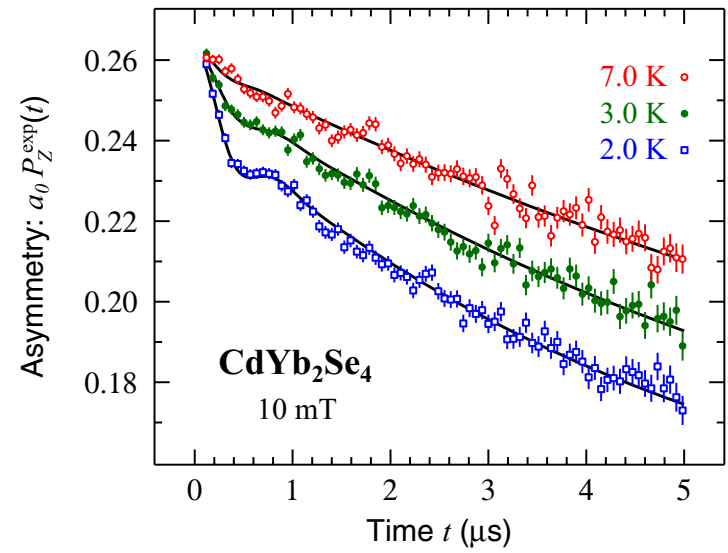

FIG. 14. $\mu$ SR spectra recorded under a $10 \mathrm{mT}$ longitudinal field in the paramagnetic phase of $\mathrm{CdYb}_{2} \mathrm{Se}_{4}$. Solid lines result from fits with the dynamical Kubo-Toyabe model. The pronounced shoulder at short times for the $2.0 \mathrm{~K}$ spectrum is a signature of the quasistatic dynamics of the magnetic field at the muon site.

behavior with a notable exception. Whereas the temperature dependence of the order parameter follows the prediction from a mean-field model based on the Brillouin function for $\mathrm{CdYb}_{2} \mathrm{~S}_{4}$, this is not the case for the selenide (Figs. 10 and 11). We find the magnetic moment and even more convincingly the spontaneous field at the muon site, which is expected to be proportional to it, to drop more slowly. This is quantitatively illustrated by the difference in the exponent $\beta$ resulting from the fits shown in the two figures. A possibility could be that the dimensionality of the magnetic interactions is reduced in the selenide compared to the sulfide. The difference must be subtle since it is not reflected in the macroscopic data reported in Sec. III A.

We now discuss the observed magnetic structures and compare them with structures already established for pyrochlore oxides. Then we shall consider the spin dynamics in the ordered state, followed by a discussion of the dynamics in the correlated paramagnetic regime.

Due to powder averaging, it is not possible from our measurements to determine which of the two basis vectors of $\Gamma_{5}$ describes the magnetic structures of the two investigated spinels [39]. ${ }^{1}$ However, we already know that we are dealing with antiferromagnetic compounds with magnetic moments perpendicular to the local threefold axis. This is in clear contrast to the pyrochlore ytterbium titanate and stannate which are splayed ferromagnets [43]. These results confirm that for a given rare-earth ion the spinel chalcogenides and pyrochlore oxides can display very different magnetic properties such as the magnetic structures. Based on the work of Ref. [24], it is tempting to attribute the difference to the CEFs. However, this approach is clearly not comprehensive since, based on the sign of the second order Stevens parameter which is the same for the $\mathrm{Er}^{3+}$ and $\mathrm{Yb}^{3+}$ ions, one would

\footnotetext{
${ }^{1}$ Electron spin resonance measurements suggest the $\mathrm{CdYb}_{2} \mathrm{~S}_{4}$ magnetic structure to be described by $\psi_{2}$ rather than $\psi_{3}$ [57]. Further neutron diffraction, e.g., in an applied magnetic field, would be welcome for a confirmation.
} 
TABLE II. Magnetic space groups associated with each mode of the different irreps for $\mathbf{k}=(000)$ structures in insulating pyrochlore magnets. The magnetic space groups have been determined using the Bilbao Crystallographic Server [44]. For reference, the crystallographic space group and the magnetic moment components at each rare-earth site are indicated. The site coordinates correspond to neighboring rare earths forming the corners of a regular tetrahedron. A dash sign means that the cell content is identical to the cell above. The prime superscript in some magnetic space groups indicates that the symmetry operation is combined with time reversal. Examples of nature realization of different magnetic structures are given in the last columns together with references. $\mathrm{Er}_{2} \mathrm{Ge}_{2} \mathrm{O}_{7}$ [18] is suggested to order according to mode $\psi_{3}$ [note (i)], while $\mathrm{Yb}_{2} \mathrm{Ge}_{2} \mathrm{O}_{7}$ orders within $\Gamma_{5}$ [19], i.e., the mode combination is unknown. The magnetic structure of $\mathrm{Gd}_{2} \mathrm{Sn}_{2} \mathrm{O}_{7}$ is described by one of the three $\Gamma_{7}$ modes. ${ }^{a}$ The two spinel compounds of the current study, $\mathrm{CdYb}_{2} \mathrm{~S}_{4}$ and $\mathrm{CdYb}_{2} \mathrm{Se}_{4}$, order according to the $\psi_{2}$ or $\psi_{3}$ mode $\left(\Gamma_{5}\right)$ or a linear combination of them. In the latter case, their magnetic space group would be $F d d d$, like their space group. Notice that the $\mathrm{Yb}_{2} \mathrm{Ti}_{2} \mathrm{O}_{7}$ magnetic structure does not belong to any of the mentioned representations. Its symmetry is lower: the magnetic space group is $I m^{\prime} m^{\prime} a$ and the corresponding structural space group is Imma [43].

\begin{tabular}{|c|c|c|c|c|c|c|c|c|c|c|c|c|c|c|c|c|}
\hline irrep & mode & & & & & & & & & & & & & $\begin{array}{l}\text { magnetic } \\
\text { group }\end{array}$ & $\begin{array}{l}\text { space } \\
\text { group }\end{array}$ & Examples or note \\
\hline & & $m_{x}$ & $\begin{array}{l}\frac{1}{2} \frac{1}{2} \frac{1}{2} \\
m_{y}\end{array}$ & $m_{z}$ & $m_{x}$ & $\begin{array}{c}\frac{1}{2} \frac{1}{4} \frac{1}{4} \\
m_{y}\end{array}$ & $m_{z}$ & $m_{x}$ & $\begin{array}{l}\frac{1}{4} \frac{1}{2} \frac{1}{4} \\
m_{y}\end{array}$ & $m_{z}$ & $m_{x}$ & $\begin{array}{l}\frac{1}{4} \frac{1}{4} \frac{1}{2} \\
m_{y}\end{array}$ & $m_{z}$ & & & \\
\hline$\Gamma_{3}$ & $\psi_{1}$ & 1 & 1 & 1 & 1 & $\overline{1}$ & $\overline{1}$ & $\overline{1}$ & 1 & $\overline{1}$ & $\overline{1}$ & $\overline{1}$ & 1 & $F d \overline{3} m^{\prime}$ & $F d \overline{3} m$ & $\begin{array}{l}\mathrm{Nd}_{2} \mathrm{Sn}_{2} \mathrm{O}_{7}[45], \mathrm{Nd}_{2} \mathrm{Zr}_{2} \mathrm{O}_{7}[3] \text {, } \\
\text { and } \mathrm{Nd}_{2} \mathrm{Hf}_{2} \mathrm{O}_{7}[10]\end{array}$ \\
\hline$\Gamma_{5}$ & $\psi_{2}$ & 1 & 1 & $\overline{2}$ & 1 & $\overline{1}$ & 2 & $\overline{1}$ & 1 & 2 & $\overline{1}$ & $\overline{1}$ & $\overline{2}$ & $I 4_{1}^{\prime} / a m^{\prime} d$ & $I 4_{1} /$ amd & $\mathrm{Er}_{2} \mathrm{Ti}_{2} \mathrm{O}_{7}[39,40]$ \\
\hline - & $\psi_{3}$ & $\overline{1}$ & 1 & 0 & $\overline{1}$ & $\overline{1}$ & 0 & 1 & 1 & 0 & 1 & $\overline{1}$ & 0 & $I 4_{1} /$ amd & - & (i) \\
\hline$\Gamma_{7}$ & $\psi_{4}$ & 1 & $\overline{1}$ & 0 & $\overline{1}$ & $\overline{1}$ & 0 & 1 & 1 & 0 & $\overline{1}$ & 1 & 0 & $I 4_{1}^{\prime} / a m d^{\prime}$ & - & \\
\hline - & $\psi_{5}$ & 0 & 1 & $\overline{1}$ & 0 & $\overline{1}$ & 1 & 0 & $\overline{1}$ & $\overline{1}$ & 0 & 1 & 1 & - & - & \\
\hline - & $\psi_{6}$ & $\overline{1}$ & 0 & 1 & 1 & 0 & 1 & 1 & 0 & $\overline{1}$ & $\overline{1}$ & 0 & $\overline{1}$ & - & - & \\
\hline$\Gamma_{9}$ & $\psi_{7}$ & 1 & 1 & 0 & $\overline{1}$ & 1 & 0 & 1 & $\overline{1}$ & 0 & $\overline{1}$ & $\overline{1}$ & 0 & $I 4_{1} / a m^{\prime} d^{\prime}$ & - & $\mathrm{Tb}_{2} \mathrm{Sn}_{2} \mathrm{O}_{7}[46,47]$ \\
\hline - & $\psi_{8}$ & 0 & 0 & 1 & 0 & 0 & 1 & 0 & 0 & 1 & 0 & 0 & 1 & - & - & and $\mathrm{Yb}_{2} \mathrm{Sn}_{2} \mathrm{O}_{7}[28,48]$ \\
\hline - & $\psi_{9}$ & 0 & 1 & 1 & 0 & $\overline{1}$ & $\overline{1}$ & 0 & $\overline{1}$ & 1 & 0 & 1 & $\overline{1}$ & - & - & \\
\hline - & $\psi_{10}$ & 1 & 0 & 0 & 1 & 0 & 0 & 1 & 0 & $\underline{0}$ & 1 & 0 & 0 & - & - & \\
\hline - & $\psi_{11}$ & 1 & 0 & 1 & $\overline{1}$ & 0 & 1 & $\overline{1}$ & 0 & $\overline{1}$ & 1 & 0 & $\overline{1}$ & - & - & \\
\hline- & $\psi_{12}$ & 0 & 1 & 0 & 0 & 1 & 0 & 0 & 1 & 0 & 0 & 1 & 0 & - & - & \\
\hline
\end{tabular}

${ }^{a}$ Notice that neutron powder diffraction, at least in zero field, does not allow to distinguish modes such as $\psi_{4}, \psi_{5}$ and $\psi_{6}$. If the actual $\mathrm{Gd}_{2} \mathrm{Sn}_{2} \mathrm{O}_{7}$ structure is a linear combination of two or three of the $\Gamma_{7}$ modes, the magnetic space group symmetry is lower than $I 4_{1}^{\prime} / a m d^{\prime}$.

expect $\mathrm{CdYb}_{2} \mathrm{Se}_{4}$ to have the same easy-axis anisotropy as $\mathrm{CdEr}_{2} \mathrm{Se}_{4}$ [24], which is not observed. In fact, the exchange interaction anisotropy could explain the difference, as for the $\mathrm{Er}_{2} \mathrm{Ti}_{2} \mathrm{O}_{7}-\mathrm{Yb}_{2} \mathrm{Ti}_{2} \mathrm{O}_{7}$ pair.

In Table II, we provide the magnetic and structural space groups corresponding to the modes associated with the four $\Gamma_{3,5,7,9}$ irreps, which are found for magnetic structures with a $\mathbf{k}=(000)$ propagation vector. In addition, we list the related rare-earth pyrochlores and spinel chalcogenides for which the magnetic structures have been determined, restricting ourselves to insulating systems where only the rare-earth sublattice is magnetic. ${ }^{2}$ Remarkably, only the neodymium oxides keep their paramagnetic cubic crystal structure as they order magnetically, thanks to the all-in-all-out magnetic structure they adopt. The diversity of magnetic structures illustrates the ground state delicate nature resulting from frustration. A consistent theoretical modeling of this large variety would be welcome. A first-step theoretical analysis has just been published [49]. It takes for granted $\mathbf{k}=(000)$ and determines the magnetic ground state as a function of Curnoe's exchange parameters [50] using a set of complementary approximate methods. A justification of the ubiquitous $\mathbf{k}=(000)$ would be

\footnotetext{
${ }^{2}$ The pyrochlore $\mathrm{Gd}_{2} \mathrm{Ti}_{2} \mathrm{O}_{7}$ is not listed because its propagation wave vector is $\mathbf{k}=(1 / 2,1 / 2,1 / 2)$. In fact, its magnetic structure is still under debate [59].
}

worthwhile. In addition, this approach is still only valid for fictitious spinor crystal-field ground states.

We now consider the $\mu \mathrm{SR}$ data in the ordered magnetic states. Spontaneous fields are observed for both compounds. However, scaling them with the spontaneous field and the magnetic moment of $\mathrm{Cr}$ reported for the chromium thio-spinel $\mathrm{CdCr}_{2} \mathrm{~S}_{4}$ [51,52], we compute an ytterbium magnetic moment $\approx 0.1 \mu_{\mathrm{B}}$. This is too small by nearly an order of magnitude. In fact, the absence of a spontaneous field in magnetically ordered pyrochlores is not a rare feature [30]. It has been argued to be the signature of the dynamical nature of the magnetic ground state [47]. It could be related to the fragmentation of the $\mathrm{Yb}^{3+}$ magnetic moments into two parts: static and dynamical components. The latter component could wash out the spontaneous field to a certain extent in the $\mu$ SR time scale. It would be complete for $\mathrm{Tb}_{2} \mathrm{Sn}_{2} \mathrm{O}_{7}$, for example, and partial for the compounds of interest in this paper. A picture within a classical physics framework has been presented [53]. An extension incorporating quantum mechanics is urgently needed.

As usual, for geometrically frustrated materials, persistent spin dynamics is present in the ordered state. This is detected through a measured temperature independent spin-lattice relaxation rate and has been taken as the signature of spin loops [26]. This is again a proof of exotic spin fluctuations in the ordered state, albeit of origin different from the anomalously small spontaneous fields. In fact, persistent spin dynamics is ubiquitous no matter the 
ground state of the compound, i.e., magnetically ordered or not.

Finally, we turn to the correlated paramagnetic regime probed up to $\approx 10 \mathrm{~K}$. Similar results are again obtained for the two compounds. The most remarkable feature is the temperature independence deep in the paramagnetic phase, up to $10 \mathrm{~K}$, of the inverse of the correlation time of the magnetic fluctuations with a value $\approx 3 \mu \mathrm{s}^{-1}$. Evidence is now accumulating for spin dynamics in this time range or even slower [31,42,54]. It could reflect a double spin-flip tunneling relaxation mechanism [55] as recently argued [54]. Interestingly, the approach to the magnetic phase transition is not seen through spin dynamics, but in the noticeable increase of the static field width as the compound is cooled. This is still to be understood.

\section{CONCLUSIONS}

An experimental study of two spinel ytterbium chalcogenides has been presented. In contrast to the splayed ferromagnetic order of the pyrochlore ytterbium stannate and titanate, the spinels are antiferromagnets with moments perpendicular to the local three-fold axis. Their magnetic structures can be described by the $\Gamma_{5}$ representation. Spontaneous magnetic fields at the muon site are observed, albeit with extremely reduced values. This suggests the magnetic (ordered) ground states to be of dynamical origin. Persistent spin dynamics observed through the spin lattice-relaxation channel are an additional proof of it. Quantum spin tunneling is detected in the correlated paramagnetic regime.
These magnetic properties invite us to consider tetrahedra of spins rather than single-ion spins as the building blocks in the correlated paramagnetic regime and its extension in the ordered states [26]. In fact, it seems that the dynamical magnetic properties of the lattice of corner-sharing tetrahedra owe much to their topology since these properties are shared by a large number of compounds, no matter the nature of their magnetic structure. Interestingly, tetrahedra of spins have recently been considered as key ingredients for a theoretical modeling of the physics in the magnetically ordered states of corner-sharing tetrahedra systems [49].

From the experimental viewpoint, a spectroscopic determination of the CEFs for the different compounds is needed to complete the experimental physical picture. Combining results from spinels with different rare-earth ions for that purpose would be of great help to pinpoint the six crystal-field parameters $[21,56]$.

\section{ACKNOWLEDGMENTS}

We are grateful to D. Ryan for discussions. This research project has been partially supported by the European Commission under the 7th Framework Programme through the "Research Infrastructures" action of the "Capacities" Programme, Contract No.: CP-CSA_INFRA-2008-1.1.1 Number 226507-NMI3. Part of this work was performed at the Institut Laue Langevin, Grenoble, France, the ISIS pulsed muon facility, STFC Rutherford Appleton Laboratory, Chilton, United Kingdom, and the Swiss muon source of the Paul Scherrer Institute, Villigen, Switzerland.
[1] J. S. Gardner, M. J. P. Gingras, and J. E. Greedan, Magnetic pyrochlore oxides, Rev. Mod. Phys. 82, 53 (2010).

[2] M. J. P. Gingras and P. A. McClarty, Quantum spin ice: A search for gapless quantum spin liquids in pyrochlore magnets, Rep. Prog. Phys. 77, 056501 (2014).

[3] E. Lhotel, S. Petit, S. Guitteny, O. Florea, M. C. Hatnean, C. Colin, E. Ressouche, M. R. Lees, and G. Balakrishnan, Fluctuations and All-in-All-Out Ordering in Dipole-Octupole $\mathrm{Nd}_{2} \mathrm{Zr}_{2} \mathrm{O}_{7}$, Phys. Rev. Lett. 115, 197202 (2015).

[4] J. Xu, V. K. Anand, A. K. Bera, M. Frontzek, D. L. Abernathy, N. Casati, K. Siemensmeyer, and B. Lake, Magnetic structure and crystal-field states of the pyrochlore antiferromagnet $\mathrm{Nd}_{2} \mathrm{Zr}_{2} \mathrm{O}_{2}$, Phys. Rev. B 92, 224430 (2015).

[5] J. Xu, C. Balz, C. Baines, H. Luetkens, and B. Lake, Spin dynamics of the ordered dipolar-octupolar pseudospin- $\frac{1}{2}$ pyrochlore $\mathrm{Nd}_{2} \mathrm{Zr}_{2} \mathrm{O}_{7}$ probed by muon spin relaxation, Phys. Rev. B 94, 064425 (2016).

[6] P. Bonville, S. Guitteny, A. Gukasov, I. Mirebeau, S. Petit, C. Decorse, M. C. Hatnean, and G. Balakrishnan, Magnetic properties and crystal field in $\mathrm{Pr}_{2} \mathrm{Zr}_{2} \mathrm{O}_{7}$, Phys. Rev. B 94, 134428 (2016).

[7] S. Petit, E. Lhotel, B. Canals, M. C. Hatnean, J. Ollivier, H. Mutka, E. Ressouche, A. R. Wildes, M. R. Lees, and G. Balakrishnan, Observation of magnetic fragmentation in spin ice, Nat. Phys. 12, 746 (2016).

[8] J.-J. Wen, S. M. Koohpayeh, K. A. Ross, B. A. Trump, T. M. McQueen, K. Kimura, S. Nakatsuji, Y. Qiu, D. M. Pajerowski,
J. R. D. Copley, and C. L. Broholm, Disordered Route to the Coulomb Quantum Spin Liquid: Random Transverse Fields on Spin Ice in $\mathrm{Pr}_{2} \mathrm{Zr}_{2} \mathrm{O}_{7}$, Phys. Rev. Lett. 118, 107206 (2017).

[9] R. Sibille, E. Lhotel, M. C. Hatnean, G. Balakrishnan, B. Fåk, N. Gauthier, T. Fennell, and M. Kenzelmann, Candidate quantum spin ice in the pyrochlore $\mathrm{Pr}_{2} \mathrm{Hf}_{2} \mathrm{O}_{7}$, Phys. Rev. B 94, 024436 (2016).

[10] V. K. Anand, A. K. Bera, J. Xu, T. Herrmannsdörfer, C. Ritter, and $\mathrm{B}$. Lake, Observation of long-range magnetic ordering in pyrohafnate $\mathrm{Nd}_{2} \mathrm{Hf}_{2} \mathrm{O}_{7}$ : A neutron diffraction study, Phys. Rev. B 92, 184418 (2015).

[11] V. K. Anand, L. Opherden, J. Xu, D. T. Adroja, A. T. M. N. Islam, T. Herrmannsdörfer, J. Hornung, R. Schönemann, M. Uhlarz, H. C. Walker, N. Casati, and B. Lake, Physical properties of the candidate quantum spin-ice system $\mathrm{Pr}_{2} \mathrm{Hf}_{2} \mathrm{O}_{7}$, Phys. Rev. B 94, 144415 (2016).

[12] C. R. Wiebe and A. M. Hallas, Frustration under pressure: Exotic magnetism in new pyrochlore oxides, APL Mater. 3, 041519 (2015).

[13] A. M. Hallas, A. M. Arevalo-Lopez, A. Z. Sharma, T. Munsie, J. P. Attfield, C. R. Wiebe, and G. M. Luke, Magnetic frustration in lead pyrochlores, Phys. Rev. B 91, 104417 (2015).

[14] Y. Q. Cai, Q. Cui, X. Li, Z. L. Dun, J. Ma, C. dela Cruz, Y. Y. Jiao, J. Liao, P. J. Sun, Y. Q. Li, J. S. Zhou, J. B. Goodenough, H. D. Zhou, and J.-G. Cheng, High-pressure synthesis and characterization of the effective pseudospin $S=$ 
1/2 XY pyrochlores $R_{2} \mathrm{Pt}_{2} \mathrm{O}_{7}(R=\mathrm{Er}, \mathrm{Yb})$, Phys. Rev. B 93, 014443 (2016).

[15] A. M. Hallas, A. Z. Sharma, Y. Cai, T. J. Munsie, M. N. Wilson, M. Tachibana, C. R. Wiebe, and G. M. Luke, Relief of frustration in the Heisenberg pyrochlore antiferromagnet $\mathrm{Gd}_{2} \mathrm{Pt}_{2} \mathrm{O}_{7}$, Phys. Rev. B 94, 134417 (2016).

[16] X. Li, Y. Q. Cai, Q. Cui, C. J. Lin, Z. L. Dun, K. Matsubayashi, Y. Uwatoko, Y. Sato, T. Kawae, S. J. Lv, C. Q. Jin, J.-S. Zhou, J. B. Goodenough, H. D. Zhou, and J.-G. Cheng, Long-range magnetic order in the Heisenberg pyrochlore antiferromagnets $\mathrm{Gd}_{2} \mathrm{Ge}_{2} \mathrm{O}_{7}$ and $\mathrm{Gd}_{2} \mathrm{Pt}_{2} \mathrm{O}_{7}$ synthesized under high pressure, Phys. Rev. B 94, 214429 (2016).

[17] Z. L. Dun, M. Lee, E. S. Choi, A. M. Hallas, C. R. Wiebe, J. S. Gardner, E. Arrighi, R. S. Freitas, A. M. ArevaloLopez, J. P. Attfield, H. D. Zhou, and J. G. Cheng, Chemical pressure effects on magnetism in the quantum spin liquid candidates $\mathrm{Yb}_{2} X_{2} \mathrm{O}_{7}(X=\mathrm{Sn}, \mathrm{Ti}, \mathrm{Ge})$, Phys. Rev. B 89, 064401 (2014).

[18] Z. L. Dun, X. Li, R. S. Freitas, E. Arrighi, C. R. Dela Cruz, M. Lee, E. S. Choi, H. B. Cao, H. J. Silverstein, C. R. Wiebe, J. G. Cheng, and H. D. Zhou, Antiferromagnetic order in the pyrochlores $R_{2} \mathrm{Ge}_{2} \mathrm{O}_{7}(R=\mathrm{Er}, \mathrm{Yb})$, Phys. Rev. B 92, 140407 (2015).

[19] A. M. Hallas, J. Gaudet, M. N. Wilson, T. J. Munsie, A. A. Aczel, M. B. Stone, R. S. Freitas, A. M. Arevalo-Lopez, J. P. Attfield, M. Tachibana, C. R. Wiebe, G. M. Luke, and B. D. Gaulin, XY antiferromagnetic ground state in the effective $S=\frac{1}{2}$ pyrochlore $\mathrm{Yb}_{2} \mathrm{Ge}_{2} \mathrm{O}_{7}$, Phys. Rev. B 93, 104405 (2016).

[20] A. M. Hallas, J. Gaudet, N. P. Butch, M. Tachibana, R. S. Freitas, G. M. Luke, C. R. Wiebe, and B. D. Gaulin, Universal dynamic magnetism in $\mathrm{Yb}$ pyrochlores with disparate ground states, Phys. Rev. B 93, 100403 (2016).

[21] A. Bertin, Y. Chapuis, P. Dalmas de Réotier, and A. Yaouanc, Crystal electric field at the rare-earth ion in the $\mathrm{R}_{2} \mathrm{Ti}_{2} \mathrm{O}_{7}$ pyrochlore compounds, J. Phys.: Condens. Matter 24, 256003 (2012).

[22] G. C. Lau, R. S. Freitas, B. G. Ueland, P. Schiffer, and R. J. Cava, Geometrical magnetic frustration in rare-earth chalcogenide spinels, Phys. Rev. B 72, 054411 (2005).

[23] A. Yaouanc, P. Dalmas de Réotier, C. Marin, and V. Glazkov, Single-crystal versus polycrystalline samples of magnetically frustrated $\mathrm{Yb}_{2} \mathrm{Ti}_{2} \mathrm{O}_{7}$ : Specific heat results, Phys. Rev. B 84, 172408 (2011).

[24] J. Lago, I. Živković, B. Z. Malkin, J. R. Fernandez, P. Ghigna, P. Dalmas de Réotier, A. Yaouanc, and T. Rojo, $\mathrm{CdEr}_{2} \mathrm{Se}_{4}$ : A New Erbium Spin Ice System in a Spinel Structure, Phys. Rev. Lett. 104, 247203 (2010).

[25] A. Legros, D. H. Ryan, P. Dalmas de Réotier, A. Yaouanc, and C. Marin, ${ }^{166} \mathrm{Er}$ Mössbauer spectroscopy study of magnetic ordering in a spinel-based potential spin-ice system: $\mathrm{CdEr}_{2} \mathrm{~S}_{4}$, J. Appl. Phys. 117, 17C701 (2015).

[26] A. Yaouanc, P. Dalmas de Réotier, A. Bertin, C. Marin, E. Lhotel, A. Amato, and C. Baines, Evidence for unidimensional low-energy excitations as the origin of persistent spin dynamics in geometrically frustrated magnets, Phys. Rev. B 91, 104427 (2015).

[27] T. Higo, K. Iritani, M. Halim, W. Higemoto, T. U. Ito, K. Kuga, K. Kimura, and S. Nakatsuji, Frustrated magnetism in the Heisenberg pyrochlore antiferromagnets $A \mathrm{Yb}_{2} X_{4}(A=$ Cd, Mg; $X=\mathrm{S}, \mathrm{Se}$ ), Phys. Rev. B 95, 174443 (2017).
[28] A. Yaouanc, P. Dalmas de Réotier, P. Bonville, J. A. Hodges, V. Glazkov, L. Keller, V. Sikolenko, M. Bartkowiak, A. Amato, C. Baines, P. J. C. King, P. C. M. Gubbens, and A. Forget, Dynamical Splayed Ferromagnetic Ground State in the Quantum Spin Ice $\mathrm{Yb}_{2} \mathrm{Sn}_{2} \mathrm{O}_{7}$, Phys. Rev. Lett. 110, 127207 (2013).

[29] A. Yaouanc, P. Dalmas de Réotier, L. Keller, B. Roessli, and A. Forget, A novel type of splayed ferromagnetic order observed in $\mathrm{Yb}_{2} \mathrm{Ti}_{2} \mathrm{O}_{7}$, J. Phys.: Condens. Matter 28, 426002 (2016).

[30] P. Dalmas de Réotier, A. Maisuradze, and A. Yaouanc, Recent $\mu \mathrm{SR}$ studies of insulating rare-earth pyrochlore magnets, J. Phys. Soc. Jpn. 85, 091010 (2016).

[31] P. Dalmas de Réotier, A. Yaouanc, A. Maisuradze, A. Bertin, P. J. Baker, A. D. Hillier, and A. Forget, Slow spin tunneling in the paramagnetic phase of the pyrochlore $\mathrm{Nd}_{2} \mathrm{Sn}_{2} \mathrm{O}_{7}$, Phys. Rev. B 95, 134420 (2017).

[32] P. Dalmas de Réotier, A. Yaouanc, P. C. M. Gubbens, C. T. Kaiser, C. Baines, and P. J. C. King, Absence of Magnetic Order in $\mathrm{Yb}_{3} \mathrm{Ga}_{5} \mathrm{O}_{12}$ : Relation Between Phase Transition and Entropy in Geometrically Frustrated Materials, Phys. Rev. Lett. 91, 167201 (2003).

[33] P. Dalmas de Réotier, A. Yaouanc, Y. Chapuis, S. H. Curnoe, B. Grenier, E. Ressouche, C. Marin, J. Lago, C. Baines, and S. R. Giblin, Magnetic order, magnetic correlations and spin dynamics in the pyrochlore antiferromagnet $\mathrm{Er}_{2} \mathrm{Ti}_{2} \mathrm{O}_{7}$, Phys. Rev. B 86, 104424 (2012).

[34] A. P. Ramirez, in Handbook of Magnetic Materials, edited by K. H. J. Buschow (Elsevier, Amsterdam, 2001), Vol. 13.

[35] L. Ben-Dor and I. Shilo, Structure and magnetic properties of sulfides of the type $\mathrm{Cd} R E_{2} \mathrm{~S}_{4}$ and $\mathrm{Mg}\left(\mathrm{Gd}_{x} \mathrm{Yb}_{1-x}\right)_{2} \mathrm{~S}_{4}$, J. Solid State Chem. 35, 278 (1980).

[36] L. Suchow and N. R. Stemple, Fluorescent rare earths in semiconducting thiospinels, J. Electrochem. Soc. 111, 191 (1964).

[37] J. Rodriguez-Carvajal, Recent advances in magnetic structure determination by neutron powder diffraction, Physica B 192, 55 (1993).

[38] A. S. Wills, M. E. Zhitomirsky, B. Canals, J. P. Sanchez, P. Bonville, P. Dalmas de Réotier, and A. Yaouanc, Magnetic ordering in $\mathrm{Gd}_{2} \mathrm{Sn}_{2} \mathrm{O}_{7}$ : The archetypal Heisenberg pyrochlore antiferromagnet, J. Phys.: Condens. Matter 18, L37-L42 (2006).

[39] A. Poole, A. S. Wills, and E. Lelièvre-Berna, Magnetic ordering in the XY pyrochlore antiferromagnet $\mathrm{Er}_{2} \mathrm{Ti}_{2} \mathrm{O}_{7}$ : A spherical neutron polarimetry study, J. Phys.: Condens. Matter 19, 452201 (2007).

[40] J. D. M. Champion, M. J. Harris, P. C. W. Holdsworth, A. S. Wills, G. Balakrishnan, S. T. Bramwell, E. Čižmár, T. Fennell, J. S. Gardner, J. Lago, D. F. McMorrow, M. Orendáč, A. Orendáčová, D. McK. Paul, R. I. Smith, M. T. F. Telling, and A. Wildes, $\mathrm{Er}_{2} \mathrm{Ti}_{2} \mathrm{O}_{7}$ : Evidence of quantum order by disorder in a frustrated antiferromagnet, Phys. Rev. B 68, 020401(R) (2003).

[41] A. Yaouanc and P. Dalmas de Réotier, Muon Spin Rotation, Relaxation, and Resonance: Applications to Condensed Matter (Oxford University Press, Oxford, 2011).

[42] A. Maisuradze, P. Dalmas de Réotier, A. Yaouanc, A. Forget, C. Baines, and P. J. C. King, Anomalously slow spin dynamics and short-range correlations in the quantum spin ice systems $\mathrm{Yb}_{2} \mathrm{Ti}_{2} \mathrm{O}_{7}$ and $\mathrm{Yb}_{2} \mathrm{Sn}_{2} \mathrm{O}_{7}$, Phys. Rev. B 92, 094424 (2015).

[43] $\mathrm{Yb}_{2} \mathrm{Ti}_{2} \mathrm{O}_{7}$ and $\mathrm{Yb}_{2} \mathrm{Sn}_{2} \mathrm{O}_{7}$ have been shown to exhibit two different variants of splayed ferromagnetic order, distinguished 
by different magnetic components perpendicular to their easy axes [28,29] although this point is disputed [58].

[44] J. M. Perez-Mato, S. V. Gallego, E. S. Tasci, L. Elcoro, G. de la Flor, and M. I. Aroyo, Symmetry-based computational tools for magnetic crystallography, Annu. Rev. Mater. Res. 45, 217 (2015).

[45] A. Bertin, P. Dalmas de Réotier, B. Fåk, C. Marin, A. Yaouanc, A. Forget, D. Sheptyakov, B. Frick, C. Ritter, A. Amato, C. Baines, and P. J. C. King, $\mathrm{Nd}_{2} \mathrm{Sn}_{2} \mathrm{O}_{7}$ : An all-in-all-out pyrochlore magnet with no divergence-free field and anomalously slow paramagnetic spin dynamics, Phys. Rev. B 92, 144423 (2015).

[46] I. Mirebeau, A. Apetrei, J. Rodríguez-Carvajal, P. Bonville, A. Forget, D. Colson, V. Glazkov, J. P. Sanchez, O. Isnard, and E. Suard, Ordered Spin Ice State and Magnetic Fluctuations in $\mathrm{Tb}_{2} \mathrm{Sn}_{2} \mathrm{O}_{7}$, Phys. Rev. Lett. 94, 246402 (2005).

[47] P. Dalmas de Réotier, A. Yaouanc, L. Keller, A. Cervellino, B. Roessli, C. Baines, A. Forget, C. Vaju, P. C. M. Gubbens, A. Amato, and P. J. C. King, Spin Dynamics and Magnetic Order in Magnetically Frustrated $\mathrm{Tb}_{2} \mathrm{Sn}_{2} \mathrm{O}_{7}$, Phys. Rev. Lett. 96, 127202 (2006).

[48] J. Lago, I. Živković, J. O. Piatek, P. Álvarez, D. Hüvonen, F. L. Pratt, M. Díaz, and T. Rojo, Glassy dynamics in the low-temperature inhomogeneous ferromagnetic phase of the quantum spin ice $\mathrm{Yb}_{2} \mathrm{Sn}_{2} \mathrm{O}_{7}$, Phys. Rev. B 89, 024421 (2014).

[49] H. Yan, O. Benton, L. Jaubert, and N. Shannon, Theory of multiple-phase competition in pyrochlore magnets with anisotropic exchange with application to $\mathrm{Yb}_{2} \mathrm{Ti}_{2} \mathrm{O}_{7}, \mathrm{Er}_{2} \mathrm{Ti}_{2} \mathrm{O}_{7}$, and $\mathrm{Er}_{2} \mathrm{Sn}_{2} \mathrm{O}_{7}$, Phys. Rev. B 95, 094422 (2017).

[50] S. H. Curnoe, Structural distortion and the spin liquid state in $\mathrm{Tb}_{2} \mathrm{Ti}_{2} \mathrm{O}_{7}$, Phys. Rev. B 78, 094418 (2008).

[51] O. Hartmann, G. M. Kalvius, R. Wäppling, A. Günther, V. Tsurkan, A. Krimmel, and A. Loid, Magnetic properties of the multiferroic chromium thio-spinels $\mathrm{CdCr}_{2} \mathrm{~S}_{4}$ and $\mathrm{HgCr}_{2} \mathrm{~S}_{4}$, Eur. Phys. J. B 86, 148 (2013).

[52] P. K. Baltzer, H. W. Lehmann, and M. Robbins, Insulating Ferromagnetic Spinels, Phys. Rev. Lett. 15, 493 (1965).

[53] M. E. Brooks-Bartlett, S. T. Banks, L. D. C. Jaubert, A. Harman-Clarke, and P. C. W. Holdsworth, Magnetic-Moment Fragmentation And Monopole Crystallization, Phys. Rev. X 4, 011007 (2014).

[54] M. Orendáč, K. Tibenská, J. Strečka, J. Čisárová, V. Tkáč, A. Orendáčová, E. Čižmár, J. Prokleška, and V. Sechovský, Cross-tunneling and phonon bottleneck effects in the relaxation phenomena of $X Y$ pyrochlore antiferromagnet $\mathrm{Er}_{2} \mathrm{Ti}_{2} \mathrm{O}_{7}$, Phys. Rev. B 93, 024410 (2016).

[55] N. Bloembergen, S. Shapiro, P. S. Pershan, and J. O. Artman, Cross-relaxation in spin systems, Phys. Rev. 114, 445 (1959).

[56] M. Ruminy, E. Pomjakushina, K. Iida, K. Kamazawa, D. T. Adroja, U. Stuhr, and T. Fennell, Crystal-field parameters of the rare-earth pyrochlores $R_{2} \mathrm{Ti}_{2} \mathrm{O}_{7}(R=\mathrm{Tb}$, Dy, and $\mathrm{H})$, Phys. Rev. B 94, 024430 (2016).

[57] D. Yoshizawa, T. Kida, S. Nakatsuji, K. Iritani, M. Halim, T. Takeuchi, and M. Hagiwara, High-field multi-frequency ESR in the rare-earth spinel compound $\mathrm{CdYb}_{2} \mathrm{~S}_{4}$, Appl. Magn. Reson. 46, 993 (2015).

[58] J. Gaudet, K. A. Ross, E. Kermarrec, N. P. Butch, G. Ehlers, H. A. Dabkowska, and B. D. Gaulin, Gapless quantum excitations from an icelike splayed ferromagnetic ground state in stoichiometric $\mathrm{Yb}_{2} \mathrm{Ti}_{2} \mathrm{O}_{7}$, Phys. Rev. B 93, 064406 (2016).

[59] J. A. M. Paddison, A. B. Cairns, D. D. Khalyavin, P. Manuel, A. Daoud-Aladine, G. Ehlers, O. A. Petrenko, J. S. Gardner, H. D. Zhou, A. L. Goodwin, and J. R. Stewart, Nature of partial magnetic order in the frustrated antiferromagnet $\mathrm{Gd}_{2} \mathrm{Ti}_{2} \mathrm{O}_{7}$, arXiv:1506.05045. 